A N N A LES

UNIVERSITATIS MARIAE CURIE-SKŁODOWSKA

LUBLIN - POLONIA

VOL. LXII, 2008

SECTIO A

$113-121$

BOŻENA PIĄTEK

\title{
Best approximation of coincidence points in metric trees
}

\begin{abstract}
In this work we present results on fixed points, pairs of coincidence points and best approximation for $\varepsilon$-semicontinuous mappings in metric trees. It is a generalization of the similar properties of upper and almost lower semicontinuous mappings.
\end{abstract}

1. Introduction. In 2007 W. Kirk and B. Panyanak showed the best approximation for upper semicontinuous set-valued mappings in a nonempty closed convex and geodesically bounded subset of a complete metric tree (see [4]). On the assumption of an almost lower semicontinuity of mappings J. Markin in [6] obtained the same result. Our main goal is to give a generalization of these theorems. Moreover, some results of the existence of coincidence points are presented.

2. Definitions. Let $(M, d)$ be a metric space and let us fix $x, y \in M$. If there is a compact interval $[a, b] \subset \mathbb{R}$ and an isometric embedding $\phi:[a, b] \rightarrow$ $M$ with $\phi(a)=x$ and $\phi(b)=y$, then the image $\phi([a, b])$ is called a metric segment and it is denoted by $[x, y]$.

The metric space $M$ is called a metric tree if the following conditions hold:

(a) for each $x, y \in M$ there is a unique metric segment $[x, y]$.

2000 Mathematics Subject Classification. 47H04, 47H10, 54C65.

Key words and phrases. Metric tree, semicontinuity, fixed points, coincidence points. 
(b) if $[x, y] \cap[y, z]=\{y\}$, then $[x, z]=[x, y] \cup[y, z]$.

The definition implies (see [3, Definition 2.1]) that

(c) for all $x, y, z \in M$ there is $w \in M$ such that $[x, z] \cap[y, z]=[w, z]$.

Let $C$ be a subset of the metric tree $M$. $C$ is called convex if $[x, y] \subset C$ for all $x, y \in C$. The set $C$ is called geodesically bounded if there is no geodesic ray in $C$, i.e., an isometric image of $[0, \infty)$. By $N_{\varepsilon}(C)\left(N_{\varepsilon}^{0}(C)\right)$ we will denote the set $\{x \in M \mid d(x, C) \leq \varepsilon\} \quad(\{x \in M \mid d(x, C)<\varepsilon\})$. Moreover, we will write shortly $N_{\varepsilon}(x)$ (or $N_{\varepsilon}^{0}(x)$ ) instead of $N_{\varepsilon}(\{x\})$ or $N_{\varepsilon}^{0}(\{x\})$.

Let $X$ be a topological space and $Y$ - a metric space. The set-valued mapping $F: X \rightarrow 2^{Y}$ with nonempty values is called almost lower semicontinuous at $x_{0} \in X$ if for each $\varepsilon>0$ there is an open neighbourhood $U \subset X$ of $x_{0}$ such that

$$
\bigcap_{x \in U} N_{\varepsilon}(F(x)) \neq \emptyset
$$

(see [6]). Let $F: X \rightarrow 2^{Y}$ be a set-valued mapping with nonempty values. $F$ is called upper semicontinuous at $x_{0} \in X$ if for each open $V \subset Y$ such that $F\left(x_{0}\right) \subset V$ there exists an open set $U \subset X$ which contains $x_{0}$ such that

$$
F(U) \subset V
$$

(see [2, Definition 1.4.1, p. 38]).

Now we introduce a weaker notion of semicontinuity. A set-valued mapping $F: X \rightarrow 2^{Y}$ with nonempty values is called $\varepsilon$-semicontinuous at $x_{0} \in X$ if for each $\varepsilon>0$ there is an open neighbourhood $U$ of $x_{0}$ such that

$$
F(x) \cap N_{\varepsilon}\left(F\left(x_{0}\right)\right) \neq \emptyset
$$

for all $x \in U$. Clearly, if $F$ is almost lower semicontinuous at $x_{0}$ and $\varepsilon$ is a positive number then there exists an open neighbourhood $U$ of $x_{0}$ with

$$
N_{\varepsilon / 3}(F(x)) \cap N_{\varepsilon / 3}\left(F\left(x_{0}\right)\right) \neq \emptyset, \quad x \in U .
$$

So for each $x \in U$ there are $z$ belonging to the set in (1), $y \in F(x)$ and $y_{0} \in F\left(x_{0}\right)$ such that

$$
d(y, z) \leq \frac{\varepsilon}{2} \quad \text { and } \quad d\left(y_{0}, z\right) \leq \frac{\varepsilon}{2} .
$$

Hence $d\left(y, F\left(x_{0}\right)\right) \leq \varepsilon$ and $F(x) \cap N_{\varepsilon}\left(F\left(x_{0}\right)\right) \neq \emptyset$. In a similar way one can check that upper semicontinuous mapping $F$ is $\varepsilon$-semicontinuous. The first of the examples (see Section 4) shows that the inverse implications are not true.

In the sequel we will need the following result due to Aksoy and Khamsi (see Lemma 3.1 and 3.2 in [1], the assumption of the boundedness is superfluous):

Proposition 1. Let $M$ be a metric tree and let $X \subset M$ be a nonempty closed convex set. Then: 
(i) for each $x \in M$ there is an projection of $x$ onto $X$, i.e., an exactly one point $P_{X}(x) \in X$ for which $d\left(x, P_{X}(x)\right)=d(x, X)$.

(ii) for any $x, y \in M$ one of the following cases is satisfied:

$$
P_{X}(x)=P_{X}(y)
$$

or

$$
d(x, y)=d\left(x, P_{X}(x)\right)+d\left(P_{X}(x), P_{X}(y)\right)+d\left(P_{X}(y), y\right) .
$$

3. Main results. We recall that for two mappings $F: X \rightarrow 2^{Y}$ and $G$ : $Y \rightarrow 2^{X}$ the composition set-valued mapping $G \circ F: X \rightarrow 2^{X}$ is defined as

$$
(G \circ F)(x)=G(F(x))=\bigcup_{y \in F(x)} G(y)
$$

(see e.g. [2, p. 37]).

Lemma 2. Let $M$ and $N$ be metric trees and let $X, Y$ be nonempty closed convex subsets of $M$ and $N$, respectively. Assume that $F$ is a set-valued mapping defined in $X$ with nonempty convex closed values in $N$. Moreover, suppose that $\bar{F}: X \rightarrow 2^{Y}$ is defined as

$$
\bar{F}(x)=F(x) \cap Y
$$

if $F(x) \cap Y \neq \emptyset$ and, otherwise, a singleton $\bar{F}(x)=\left\{P_{Y}(y)\right\}$ for some point $y \in F(x)$.

If $F$ is $\varepsilon$-semicontinuous then $\bar{F}$ is also and

$$
d(z, F(x))=\inf _{y \in Y} d(y, F(x))
$$

for all $z \in \bar{F}(x)$ and $x \in X$.

Proof. At first we will show that $\bar{F}$ is well defined. Assume that $F(x) \cap Y=$ $\emptyset$ and $u, v \in F(x)$. By (ii) of Proposition 1 we have $P_{Y}(u)=P_{Y}(v)$ or $[u, v]=\left[u, P_{Y}(u)\right] \cup\left[P_{Y}(u), P_{Y}(v)\right] \cup\left[P_{Y}(v), v\right]$, but the second equality is impossible since otherwise $\left[P_{Y}(u), P_{Y}(v)\right] \subset F(x)$ and then $F(x) \cap Y \neq \emptyset$.

So $\bar{F}(x)$ is a closed convex set for each $x \in X$ and clearly (2) is satisfied. Moreover, we have that

$$
\bar{F}(x)=\left\{P_{Y}(y): y \in F(x)\right\} .
$$

Now we will prove that if $F$ is $\varepsilon$-semicontinuous, then so is $\bar{F}$. Let us take $x_{0} \in X$ and $\varepsilon>0$. There is a neighbourhood $U$ of $x_{0}$ such that for each $x \in U$ one can find $z \in F(x)$ for which

$$
d\left(z, F\left(x_{0}\right)\right) \leq \varepsilon
$$

But it implies that $d\left(P_{Y}(z), \bar{F}\left(x_{0}\right)\right) \leq \varepsilon$, which completes the proof. 
The above definition of the mapping $\bar{F}$ is equivalent to the Markin definition

$$
\bar{F}(x)=X \cap N_{\varepsilon}(F(x)), \quad \varepsilon=\inf \left\{t>0: X \cap N_{t}(F(x)) \neq \emptyset\right\}
$$

(see [6, proof of Theorem 4.4]), but it is more useful in the sequel. Moreover, by the proof of the same theorem we obtain that the almost lower semicontinuity of $F$ implies the same property of $\bar{F}$. On the assumption that $F$ is upper semicontinuous, the upper semicontinuity of $\bar{F}$ follows from the proof of [4, Theorem 2.1, p. 684].

Lemma 3. Let $M, N$ be complete metric trees and let $F: M \rightarrow 2^{N}$, $G: N \rightarrow 2^{M}$ be set-valued mappings with nonempty convex closed values. Moreover, suppose that $F$ is $\varepsilon$-semicontinuous with compact values and $G$ is upper semicontinuous. Then $G \circ F: M \rightarrow 2^{M}$ is an $\varepsilon$-semicontinuous mapping with nonempty convex closed values.

Proof. First we will prove that the mapping $G \circ F$ is $\varepsilon$-semicontinuous. For every $w \in F\left(x_{0}\right)$ we may find $\delta_{w}>0$ such that $G(y) \subset N_{\varepsilon}^{0}(G(w))$ for all $y \in N_{\delta_{w}}(w)$. Setting $U=\bigcup_{w \in F\left(x_{0}\right)} N_{\delta_{w}}^{0}(w)$ we obtain an open set $U$ containing $F\left(x_{0}\right)$ such that

$$
G(y) \subset N_{\varepsilon}^{0}\left(G\left(F\left(x_{0}\right)\right)\right), \quad y \in U .
$$

Since $F\left(x_{0}\right)$ is a compact set, there is $\delta>0$ with $N_{\delta}\left(F\left(x_{0}\right)\right) \subset U$. By the $\varepsilon$-semicontinuity of $F$ one can choose an open neighbourhood $V$ of $x_{0}$ for which

$$
F(x) \cap N_{\delta}\left(F\left(x_{0}\right)\right) \neq \emptyset, \quad x \in V .
$$

Therefore if $x \in V$ there is $y \in F(x) \cap N_{\delta}\left(F\left(x_{0}\right)\right)$. Since $y \in U$ we have $(G \circ F)(x) \cap N_{\varepsilon}\left(G \circ F\left(x_{0}\right)\right) \neq \emptyset$ by $(3)$.

To show the closedness of $G \circ F(x), x \in X$, let us take a sequence $\left(u_{n}\right)$ for which $u_{n} \in G \circ F(x)$ and $u_{n} \rightarrow u$. We have to show that $u \in G \circ F(x)$. Obviously one can find a sequence $\left(y_{n}\right), y_{n} \in F(x)$, such that $u_{n} \in G\left(y_{n}\right)$. We may assume that $y_{n} \rightarrow y, y \in F(x)$. By Proposition 1.4.8 in [2] the mapping $G$ is closed, so $u \in G(y) \subset G \circ F(x)$.

Finally we will prove the convexity of $G \circ F(x), x \in X$. Assume that there are $u, v \in G \circ F(x)$ and $w \in[u, v]$ which does not belong to $G \circ F(x)$. According to the closedness of $G \circ F(x)$ one can choose $\varepsilon>0$ such that

$$
N_{2 \varepsilon}(w) \cap G \circ F(x)=\emptyset .
$$

Let $s, t \in F(x)$ be chosen in such a way that $u \in G(s)$ and $v \in G(t)$. For each $y \in[s, t]$ one can find a number $\delta(y)>0$ for which

$$
\bigcup_{z \in N_{\delta(y)}(y)} G(z) \subset N_{\varepsilon}(G(y)) .
$$


By the compactness of the metric segment $[s, t]$ it follows that there is a finite set $\left\{y_{1}, \ldots, y_{n}\right\}$ such that

$$
[s, t] \subset \bigcup_{k=1}^{n} N_{\delta\left(y_{k}\right)}\left(y_{k}\right) .
$$

According to (5) and (6) we have

$$
\bigcup_{z \in[s, t]} G(z) \subset \bigcup_{k=1}^{n} N_{\varepsilon}\left(G\left(y_{k}\right)\right)=: U .
$$

Now we will show that $U$ is a convex set.

Without loss of generality we may assume that

$$
N_{\delta\left(y_{1}\right)}\left(y_{1}\right) \cap N_{\delta\left(y_{2}\right)}\left(y_{2}\right) \neq \emptyset \text {. }
$$

Let $p, q \in N_{\varepsilon}\left(G\left(y_{1}\right)\right) \cup N_{\varepsilon}\left(G\left(y_{2}\right)\right)$. Let us choose a $z$ belonging to the set in (7). Obviously $G(z) \subset N_{\varepsilon}\left(G\left(y_{1}\right)\right) \cap N_{\varepsilon}\left(G\left(y_{2}\right)\right)$. Assume that $r \in G(z)$. It is clear that $[p, q] \subset[p, r] \cup[q, r] \subset N_{\varepsilon}\left(G\left(y_{1}\right)\right) \cup N_{\varepsilon}\left(G\left(y_{2}\right)\right)$. By induction $U$ is convex and it follows that

$$
w \in \bigcup_{k=1}^{n} N_{\varepsilon}\left(G\left(y_{k}\right)\right) .
$$

So there is $y_{k} \in F(x)$ such that $d\left(w, G\left(y_{k}\right)\right) \leq \varepsilon$ which contradicts (4).

The assumption of the compactness of $F(x)$ is an essential condition of the closedness of values of $G \circ F$ (see Example 10).

Theorem 4. Let $M$ be a complete metric tree and let $X$ be a nonempty convex closed and geodesically bounded subset of $M$. If $F: X \rightarrow 2^{X}$ is an $\varepsilon-$ semicontinuous set-valued mapping with nonempty convex closed values then $F$ has a fixed point.

Proof. The first part of the proof is similar to the proof of Theorem 2.1 in [4]. Let $x \in X$ and let $r(x)=P_{F(x)}(x)$. If $x$ is not a fixed point, then $d(x, r(x))>0$. For each $t \in[x, r(x)]$ we define $\xi(t)$ as

$$
[x, r(x)] \cap[x, r(t)]=[x, \xi(t)] .
$$

Let

$$
\begin{gathered}
A=\{t \in[x, r(x)] \mid \xi(t) \in[x, t]\}, \\
B=\{t \in[x, r(x)] \mid \xi(t) \in[t, r(x)]\} .
\end{gathered}
$$

Clearly $r(x) \in A$ and $x \in B$. The sets $A$ and $B$ are closed. Indeed, let $\left(t_{n}\right)$ be a sequence of elements of $B$ such that $t_{n} \rightarrow t$. Assume that $t \in A \backslash B$. Then $d(t, \xi(t))=\varepsilon>0$. Let $n \in \mathbb{N}$ be such that $d\left(t, t_{n}\right)<\varepsilon / 2$. For each $u \in F(t)$ and $v \in F\left(t_{n}\right)$ we obtain

$$
r(t) \in[u, \xi(t)], \xi(t) \in\left[r(t), \xi\left(t_{n}\right)\right], \xi\left(t_{n}\right) \in\left[\xi(t), r\left(t_{n}\right)\right], r\left(t_{n}\right) \in\left[\xi\left(t_{n}\right), v\right] .
$$


Then we have

$$
\left[\xi(t), \xi\left(t_{n}\right)\right] \subset[u, v]
$$

and finally $\inf _{z \in F\left(t_{n}\right)} d(z, F(t)) \geq d\left(\xi(t), \xi\left(t_{n}\right)\right)>\varepsilon / 2$ for each $n \in \mathbb{N}$ sufficiently large which contradicts the $\varepsilon$-semicontinuity of $F$. The proof for $A$ is similar.

Since $A$ is compact there is $\varphi(x) \in[x, r(x)]$ such that $d(x, \varphi(x))=$ $\inf _{t \in A} d(x, t)$. Moreover, $\varphi(x) \in A \cap B$, which implies that

$$
[x, r(x)] \cap[x, r(\varphi(x))]=[x, \varphi(x)] .
$$

Now suppose that $F$ has not a fixed point in $X$. Therefore, we have

$$
d(x, \varphi(x))>0, \quad x \in X .
$$

Let us choose any $x_{0} \in X$. We define a transfinite sequence $\left(x_{\alpha}\right)_{\alpha<\Omega}$ such that

$$
d\left(x_{0}, x_{\alpha}\right)=\sum_{\beta<\alpha} d_{\beta}
$$

and

$$
d\left(x_{0}, \varphi\left(x_{\alpha}\right)\right)=d\left(x_{0}, x_{\alpha}\right)+d\left(x_{\alpha}, \varphi\left(x_{\alpha}\right)\right)
$$

where $\Omega$ is the order type of the set $\left\{\alpha \mid \bar{\alpha} \leq \aleph_{0}\right\}$ (see [5, p. 20-21]) and $d_{\beta}=d\left(x_{\beta}, \varphi\left(x_{\beta}\right)\right)$.

Let $\alpha$ be a limit number. By the geodesically boundedness of $X$ and (10) the countable sum $\sum_{\beta<\alpha} d_{\beta}$ is bounded. So there is a sequence of points $x_{\alpha_{n}}$ such that $\lim _{n \rightarrow \infty} \sum_{\beta<\alpha_{n}} d_{\beta}=\sum_{\beta<\alpha} d_{\beta}$ and $x_{\alpha_{n}} \rightarrow \bar{x} \in X$. Let us define $x_{\alpha}:=\bar{x}$. Clearly (10) and (11) are satisfied. The proof of (11) is not different from the proof of the closedness of $B$.

If $\alpha=\beta+1$ we define $x_{\alpha}:=\varphi\left(x_{\beta}\right)$. By (8) with $x=x_{\beta}$, (10) and (11) we obtain $d\left(x_{0}, x_{\alpha}\right)=d\left(x_{0}, x_{\beta}\right)+d\left(x_{\beta}, x_{\alpha}\right)=\sum_{\gamma<\alpha} d_{\gamma}$ and $d\left(x_{0}, \varphi\left(x_{\alpha}\right)\right)=$ $d\left(x_{0}, x_{\alpha}\right)+d_{\alpha}$.

Now let us define

$$
m:=\sup _{\alpha<\Omega} \sum_{\beta<\alpha} d_{\beta} .
$$

If $m$ were equal to the infinity, points $x_{\alpha}$ would lie on the geodesic ray. Hence $m<\infty$ and one can find a sequence $\alpha_{n}$ for which $d\left(x_{0}, x_{\alpha_{n}}\right) \rightarrow m$. Clearly there is $\alpha<\Omega$ such that $\alpha_{n}<\alpha$ for each $n \in \mathbb{N}$. Moreover, $d\left(x_{0}, x_{\alpha}\right)=m$ what implies that $d\left(x_{0}, x_{\alpha+1}\right)=d\left(x_{0}, \varphi\left(x_{\alpha}\right)\right)>m$. This contradicts (12).

Theorem 5. Let $M$ and $X$ be defined as in Theorem 4. Moreover, suppose that $F: X \rightarrow 2^{M}$ is an $\varepsilon$-semicontinuous mapping with nonempty closed convex values. Then there is a point $\bar{x} \in X$ for which

$$
d(\bar{x}, F(\bar{x}))=\inf _{x \in X} d(x, F(\bar{x})) .
$$


Proof. Let us define $\bar{F}$ in the same way as in Lemma 2, i.e., $\bar{F}(x)=F(x) \cap$ $X$ if $F(x) \cap X \neq \emptyset$, and, otherwise, $\bar{F}(x)=\left\{P_{X}(y)\right\}, y \in F(x), x \in X$. Then the assumptions of Theorem 4 hold for $\bar{F}: X \rightarrow 2^{X}$. Hence there is $\bar{x} \in X$ such that $\bar{x} \in \bar{F}(\bar{x})$.

Let us consider the set $\bar{F}(\bar{x})$. If $F(\bar{x}) \cap X \neq \emptyset$, then $d(\bar{x}, F(\bar{x}))=0=$ $\inf _{x \in X} d(x, F(\bar{x}))$ what completes the proof. Otherwise, $\{\bar{x}\}=\bar{F}(\bar{x})$ and

$$
d(\bar{x}, F(\bar{x}))=\inf _{x \in X} \inf _{y \in F(\bar{x})} d(x, y)=\inf _{x \in X} d(x, F(\bar{x})) .
$$

Since each almost lower semicontinuous or upper semicontinuous mapping is $\varepsilon$-semicontinuous, we obtain the following:

Corollary 6 ([6, Theorem 4.4] and [4, Theorem 2.1]). Let $M$ and $X$ be defined as in Theorem 5. Moreover, suppose that $F: X \rightarrow 2^{M}$ is an almost lower semicontinuous (upper semicontinuous) mapping with nonempty closed convex values. Then there is a point of the best approximation, i.e., $\bar{x} \in X$ such that (13) holds.

Theorem 7. Let $M, N$ be complete metric trees, let $X, Y$ be nonempty closed convex and geodesically bounded subsets of $M$ and $N$, respectively, and let $F: X \rightarrow 2^{N}, G: Y \rightarrow 2^{M}$ be set-valued mappings with nonempty closed convex values. Moreover, suppose that $F$ is a $\varepsilon$-semicontinuous mapping with compact values and $G$ is upper semicontinuous. Then there is a pair $(\bar{x}, \bar{y}) \in X \times Y$ of points with

$$
d_{N}(\bar{y}, F(\bar{x}))=\inf _{y \in Y} d_{N}(y, F(\bar{x})), \quad d_{M}(\bar{x}, G(\bar{y}))=\inf _{x \in X} d_{M}(x, G(\bar{y})) .
$$

Proof. Let $\bar{F}: X \rightarrow 2^{Y}$ and $\bar{G}: Y \rightarrow 2^{X}$ be projections defined in the same way as in Lemma 2. By Lemma 3 the product $\bar{G} \circ \bar{F}: X \rightarrow 2^{X}$ is a $\varepsilon$-semicontinuous mapping with nonempty closed convex values in $X$. According to Theorem 4 one can find a fixed point $\bar{x} \in X$ of $\bar{G} \circ \bar{F}$. It follows that there is $\bar{y} \in Y$ such that

$$
\bar{y} \in \bar{F}(\bar{x}) \quad \text { and } \quad \bar{x} \in \bar{G}(\bar{y}) .
$$

The rest of the proof goes in the same way as in Theorem 5 .

The last example (see Example 11) shows that it is not sufficient in Theorem 7 to assume that the mappings $F$ and $G$ are $\varepsilon$-semicontinuous. In that case a pair of best approximation does not have to exist.

Theorem 8. Let the sets $M, N, X$ and $Y$ be defined as in Theorem 7 and let $F: X \rightarrow 2^{N}, G: Y \rightarrow 2^{M}$ be set-valued mappings with nonempty closed convex values. Moreover, suppose that $F$ is $\varepsilon$-semicontinuous and $G$ is almost lower semicontinuous. Then there exists the best approximation of coincidence points, i.e., a pair $(\bar{x}, \bar{y}) \in X \times Y$ for which (14) holds. 
Proof. Let us define $\bar{F}: X \rightarrow 2^{Y}$ and $\bar{G}: Y \rightarrow 2^{X}$ in the same way as above. According to [6, Theorem 4.3] there is a continuous selection $g: Y \rightarrow X$ of $\bar{G}$. Clearly the product $\bar{F} \circ g$ is an $\varepsilon$-semicontinuous set-valued mapping with nonempty closed convex values. By Theorem 4 there exists a fixed point $\bar{y}$ of $\bar{F} \circ g$. The rest of the proof runs as before in Theorem 5 .

\section{Examples.}

Example 9. Let $F: \mathbb{R} \rightarrow 2^{\mathbb{R}}$ be defined as follows

$$
F(x)= \begin{cases}{[-1,0],} & x<0 \\ {[0,1],} & x=0 \\ {[1,2],} & x>0\end{cases}
$$

Then the mapping $F$ is $\varepsilon$-semicontinuous at $x=0$ while $F$ is not upper semicontinuous nor almost lower semicontinuous.

Example 10. Let us consider the space $\mathbb{R}^{2}$ with a radial metric $d$, i.e.,

$$
d\left(\left(x_{1}, y_{1}\right),\left(x_{2}, y_{2}\right)\right)= \begin{cases}\sqrt{\left(x_{1}-x_{2}\right)^{2}+\left(y_{1}-y_{2}\right)^{2}}, & x_{1} y_{2}=x_{2} y_{1} \\ \sqrt{x_{1}^{2}+y_{1}^{2}}+\sqrt{x_{2}^{2}+y_{2}^{2}}, & \text { otherwise }\end{cases}
$$

and suppose that $F((0,0))=N_{1}((0,0))$ which is noncompact.

A mapping $G: N_{1}((0,0)) \rightarrow 2^{\mathbb{R}^{2}}$ is defined as

$$
G(x, y)= \begin{cases}{\left[(0,0),\left(0,1-\frac{1}{n}\right)\right],} & (x, y)=\left(\cos \frac{\pi}{n}, \sin \frac{\pi}{n}\right) \\ \{(0,0)\}, & \text { otherwise. }\end{cases}
$$

Hence

$$
G \circ F((0,0))=\bigcup_{(x, y) \in N_{1}((0,0))} G(x, y)=\left\{(0, y) \in \mathbb{R}^{2} \mid 0 \leq y<1\right\} .
$$

Example 11. Let us consider the space $\mathbb{R}$ with standard metric. Let $F(1)$ be defined by

$$
F(1)=[0,1]
$$

while $G:[0,1] \rightarrow \mathbb{R}$ is defined as

$$
G(x)= \begin{cases}{[0, x],} & 0 \leq x<1 \\ \{0\}, & x=1\end{cases}
$$

Therefore $d(1, G \circ F(1))=0$ but there is not a pair of the best approximation because for each $x \in F(1)$ we have $d(1, G(x))>0$. 


\section{REFERENCES}

[1] Aksoy, A. G., Khamsi, M. A., A selection theorem in metric trees, Proc. Amer. Math. Soc. 134 (2006), 2957-2966.

[2] Aubin, J.-P., Frankowska, H., Set-valued Analysis, Birkhäuser Boston, Inc., Boston, MA, 1990

[3] Espínola, R., Kirk, W. A., Fixed point theorems in $\mathbb{R}$-trees with applications to graph theory, Topology Appl. 153 (2006), 1046-1055.

[4] Kirk, W. A., Panyanak, B., Best approximation in $\mathbb{R}$-trees, Numer. Funct. Anal. Optim. 28 (2007), 681-690.

[5] Kuczma, M., An Introduction to the Theory of Functional Equations and Inequalities. Cauchy's Equation and Jensen Inequality, Uniwersytet Śląski, Katowice, Państwowe Wydawnictwo Naukowe (PWN), Warszawa, 1985.

[6] Markin, J. T., Fixed points, selections and best approximation for multivalued mappings in R-trees, Nonlinear Anal. 67 (2007), 2712-2716.

Bożena Piątek

Institute of Mathematics

Silesian University of Technology

ul. Kaszubska 23

44-100 Gliwice, Poland

e-mail: b.piatek@polsl.pl

Received May 5, 2008 